\title{
Programação dinâmica aplicada à redução de custos nas compras de vacinas de um hospital
}

\author{
Dynamic programming applied to cost reduction in purchases of vaccines of \\ a hospital \\ Rafaela Boeira Cechin ${ }^{1}$ (D), Rodrigo Biasuz ${ }^{2}$ (D), Asdrubal Falavigna ${ }^{3}$ (D), Leandro Luís Corso ${ }^{3}$ (D)
}

\begin{abstract}
RESUMO
Uma organização não depende somente do quanto ela fatura com seus produtos e serviços, mas também do quanto ela gasta, por isso, redução de custos é tão importante, podendo até ser o diferencial para uma empresa se manter no mercado. Este artigo apresenta um estudo de caso de Programação Dinâmica aplicada a compras de vacina em um hospital, a fim de otimizar a maneira que é realizada as compras e reduzir seus custos. Para isso, foram coletadas informações relacionadas a aquisição da mercadoria e estoque. O resultado obtido com o modelo de otimização foi comparado com o cenário atual do hospital, e foi observada uma possível redução de 5,57\%, representando quase $\mathrm{R} \$ 100.000,00$.
\end{abstract}

Palavras-chave: Programação Dinâmica; Saúde; Gestão; Redução de Custos.

\begin{abstract}
An organization does not only depend on how much it makes from its products and services but also how much it spends, so cost reduction is so important and may even be the differential for a company to stay in the market. This article presents a Dynamic Programming case study on vaccine procurement in a hospital to optimize the way purchases are made and reduce costs. For this, information related to the acquisition of the merchandise and stock was collected. The result obtained with the optimization model was compared to the current hospital scenario, and a possible reduction of $5.57 \%$ was observed, representing almost $R \$ 100,000.00$.
\end{abstract}

Keywords: Dynamic Programming; Health; Management; Cost Savings.

1. Mestrado em Engenharia Mecânica. Universidade de Caxias do Sul (UCS), Caxias do Sul (RS), Brasil.

2. Engenheiro de Produção. UCS, Caxias do Sul (RS), Brasil.

3. Professor. UCS, Caxias do Sul (RS), Brasil.

$\square$ Leandro Luís Corso. Rua Francisco Getúlio Vargas, 1130. CEP 95070-560. Caxias do Sul (RS), Brasil. Ilcorso@ucs.br, leandro.Icorso@gmail.com | Recebido: 17/03/2019 | Aprovado: 09/09/2019 


\section{INTRODUÇÃO}

Um hospital pode ser analisado como uma empresa, e como tal, deve-se considerar ferramentas de gestão em sua estratégia gerencial para garantir a otimização dos investimentos, sem diminuir a qualidade do atendimento aos pacientes ${ }^{1}$. Desta forma, pode-se mencionar conhecimentos da área da saúde associados com conceitos de finança e logística 2,3,4. Abbas ${ }^{5}$ comenta em seu estudo esta visão multidisciplinar, que com o advento da tecnologia, pode auxiliar na resolução de problemas ou na otimização de processos administrativos e recursos financeiros. Já Duarte et al. ${ }^{1}$ ressaltam a importância que o processo de compras tem no papel estratégico do hospital, por ser relevante para melhorar a administração da organização quanto aos recursos escassos.

Otimizar um processo consiste em determinar o maior ou menor de valor de uma função, como, por exemplo, maximizar a capacidade de atendimento ou minimizar os custos. Com este propósito, este trabalho utilizou Programação Dinâmica (PD), que é um método para otimizar processos, em que decompõe o processo principal em diversos estágios, e a solução de forma sequencial destes estágios leva à solução do processo original.

$O$ conceito da PD pode ser utilizado em diversos conteúdos, como é o exemplo de Raupp, Gibbon e Beuren ${ }^{6}$, que aplicaram com o objetivo de maximizar a produção, considerando diversas restrições na capacidade produtiva de uma empresa de cobertores e mantas. Já Scarcellip utilizou PD em uma nova abordagem para o planejamento da operação de sistemas hidrotérmicos de potência, de forma que as usinas hidrelétricas pudessem ser representadas e otimizadas individualmente. Wang et al. ${ }^{8}$ se aprofundaram na aplicabilidade da PD em big data, apresentando um algoritmo com o objetivo de simplificar a programação paralela.

Já Diban et al. ${ }^{9}$ apresentaram um método de PD para obter uma política ótima de replantio, que tenha emissão mínima de $\mathrm{CO}_{2}$ em um horizonte de tempo finito, para plantações agrícolas comerciais. Zhang, Wu e Zuo ${ }^{10}$ propuseram um algoritmo baseado em PD para obter a solução na minimização de combustível de um gasoduto em estado transitório. Duchaud et al. ${ }^{11}$ publicaram um algoritmo de PD que minimiza a energia comprada da rede, a fim de otimizar a comercialização de energia e gerenciar o estado de carga do Sistema de Armazenamento de Energia sob várias restrições.

O objetivo deste trabalho é aplicar PD para minimizar os custos de aquisição de vacinas de um hospital, otimizando a maneira que é realizada estas compras.

\section{Referencial teórico}

A PD pode ser conceituada como um modelo matemático que fragmenta um processo em estágios mais simples, resolvendo-os sequencialmente e não de maneira recursiva ${ }^{6,8,9,11}$. Deve-se encontrar a melhor solução para todas as instâncias dos estágios, e combinando estas respostas, é obtido o resultado do processo original. Esta técnica foi desenvolvida por Richard Bellman, na década de 1950, e não possui uma formulação matemática padronizada, ou seja, as equações que descrevem o comportamento do processo são desenvolvidas para se adequarem a cada caso ${ }^{12}$.

Há duas particularidades que um processo deve ter para tornar a lógica da PD ser aplicável: subestrutura ótima e estágios sobrepostos ${ }^{8}$. Subestrutura ótima representa que a solução do processo global pode ser obtida pela combinação de soluções ótimas para seus estágios; enquanto a segunda característica significa que qualquer algoritmo recursivo que otimize o processo deve resolver os mesmos estágios repetidamente, sem decompô-los em novos estágios.

Ainda, há duas classificações para um modelo de PD: determinístico ou probabilístico6,13. A primeira é para as situações em que as variáveis de estado são discretas e o período de otimização é finito, exemplificado pelos autores como otimização relacionada as engenharias e ciências sociais; enquanto nos modelos probabilísticos existe uma distribuição de probabilidade que influencia à tomada de decisão para o estágio seguinte.

Um modelo de PD se caracteriza por iniciar em um estágio $t$ qualquer, um estado de entrada $S_{0}$, estados de saída $S_{t}$, variável de decisão $X_{t}$ que influencia na saída e no custo, um custo do estágio $f_{t}\left(e_{t-1}, x_{t}\right)$ que é influenciado também pela demanda 
$d_{t^{\prime}}$ e a transformação $\varphi_{t}$ do estágio $t^{7,10,12}$. O custo $f_{t}\left(e_{t-1}, x_{t}\right)$ mede a eficiência da transformação das entradas em saídas, e $\varphi_{t}$ expressa as saídas como uma função das entradas, isto é, $S_{t}=\varphi_{t}\left(S_{t-1}, x_{t}\right)$. A Figura 1 mostra esquematicamente a generalização do conceito para $T$ estágios.

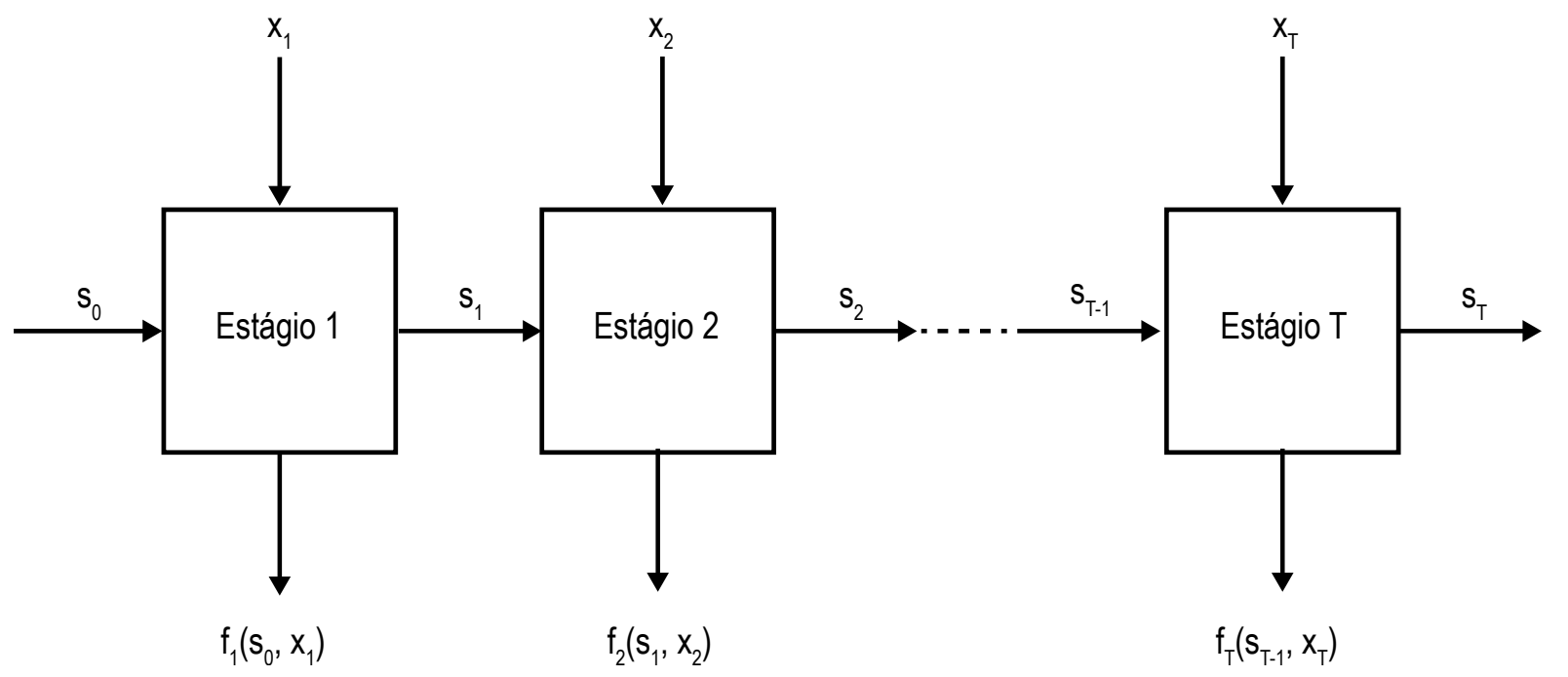

Figura 1: Caso genérico para T estágios

Fonte: Adaptado de Colin ${ }^{12}$

As equações de continuidade de fluxo e o cálculo do custo são exibidas nas Equações 1 e 2, respectivamente ${ }^{7,10,13}$.

$e_{t}=e_{t-1}+x_{t}-d_{t}$

max ou min $f_{t}\left(e_{t-1}, x_{t}\right)=\max$ ou miv [(custo do estado $\left.S_{t-1}\right)+$ (cuto viável de decisão $x_{t}$ ) + (custo de estado $\left.S_{t+1}\right)$ ]

\section{MÉTODO}

O presente método teve a finalidade de estruturar um modelo de PD que pudesse gerar a otimização dos recursos do investimento necessário para compra de vacinas. Para isso, o trabalho se dividiu em três etapas apresentadas na Figura 2.
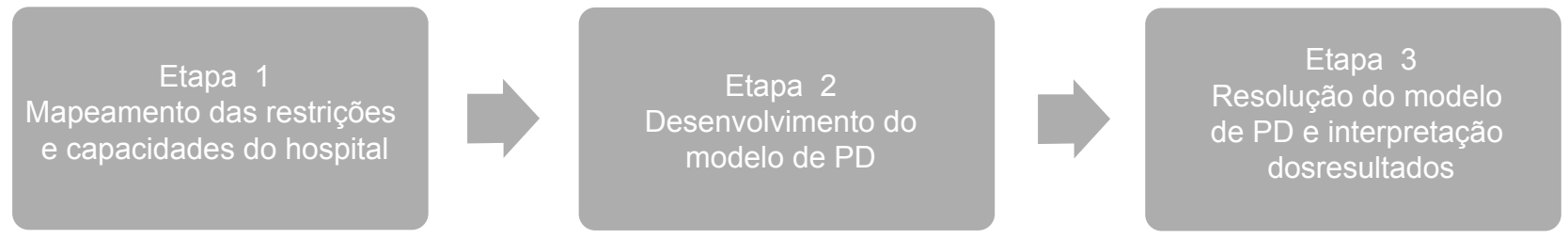

Figura 2: Etapas utilizadas para o desenvolvimento do trabalho Fonte: Elaborado pelos autores (2019)

Na Etapa 1 foram consultados os dados do hospital, que é localizado na região sul do Brasil. Para isso, os custos relativos à compra e estoque deste medicamento foram analisados. Nesta fase, se buscou relacionar as seguintes informações: capacidade física da área de estoque, estoque ini- cial, demandas mensais de vacinas, preço a ser pago pelas vacinas e custo de entrega das vacinas. Para a Etapa 2, as informações previamente listadas foram relacionadas com os parâmetros da Programação Dinâmica, conforme exibido na Figura 3. 


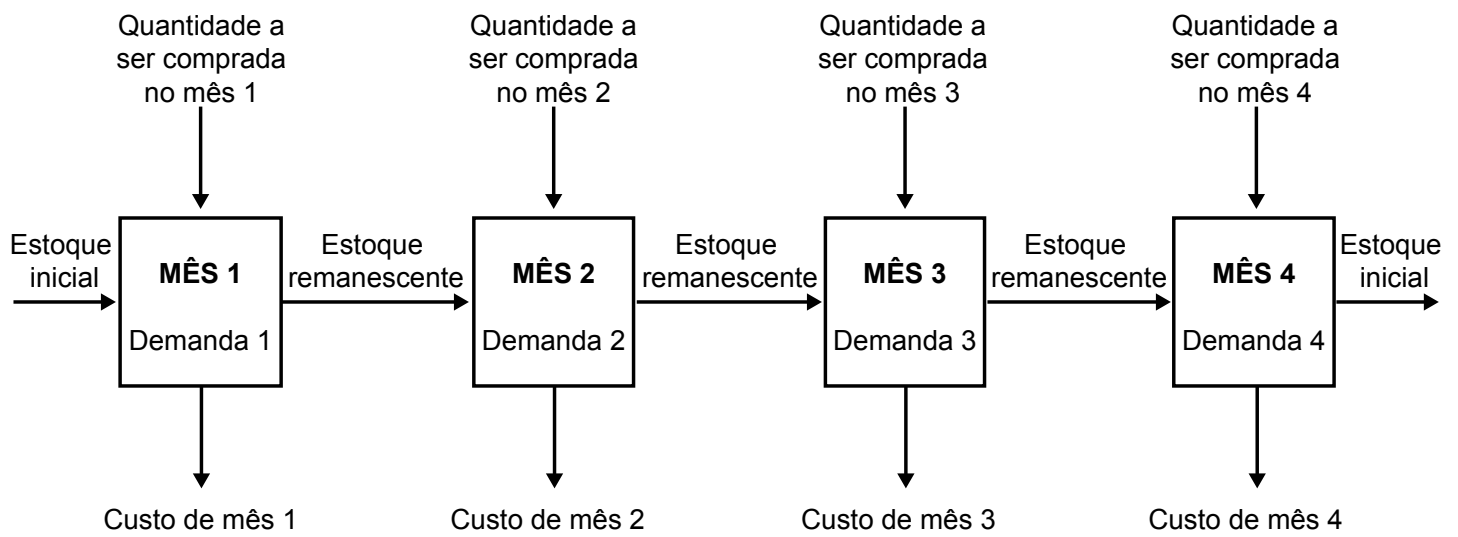

Figura 3: Dados do modelo relacionados com a Programação Dinâmica

Fonte: Elaborado pelos autores (2019)

A Figura 3 representa o esquema sequencial de como as decisões são tomadas, e observando-a, é entendido que o estoque inicial é um estado de entrada $e_{o}$ os estoques remanescentes são estados de saída $e_{t^{\prime}}$ a quantidade a ser comprada é uma variável de decisão $x_{t}$, e que o custo do mês $t$ é $f_{t}\left(e_{t-1}, x_{t}\right)$, que é influenciado também pela demanda $d_{t}$.

Para a Etapa 3, para solução do modelo criado na etapa anterior, se verificou que, em análises nos algoritmos desenvolvidos da PD e pela praticidade comprovada por testes em computadores realizados na década de 1980, o cálculo ocorre de trás para frente, assim como Diban et al. ${ }^{9}$ implementaram em seu trabalho. Portanto, para o processo em questão, o cálculo iniciou-se no mês 4, depois foi calculado o terceiro mês, em seguida o mês 2 , e finalizando no primeiro mês.

\section{RESULTADOS}

Seguindo o fluxograma apresentado na seção anterior, foram analisados os dados do hospital, para este caso se analisou os custos de quatro meses relacionados a compra e estoque deste medicamento. Os resultados da etapa de levantamento de dados resultaram nas seguintes informações:

a. o estoque é limitado por restrição física de 60.000 unidades;

b. estoque inicial deste estudo é de 5.000 unidades;

c. as demandas mensais são de $20.000,10.000,15.000$ e 10.000 unidades, respectivamente para cada mês; d. por determinação da empresa, não devem sobrar vacinas em estoque no quarto mês;

e. no primeiro mês, o custo de entrega do pedido é de $\mathrm{R} \$ 1.100,00$, porém há um aumento para $\mathrm{R} \$ 1.200,00$ nos demais meses, independentemente da quantidade do pedido;

f. a empresa fabricante da vacina comercializa lotes mínimos de 5.000 unidades, e apenas lotes múltiplos deste valor podem ser comprados;

g. o custo de estoque é de $1,85 \%$ do valor total mensal do estoque para os dois primeiros meses, e de $2 \%$ para os dois seguintes;

h. o preço da vacina é de $\mathrm{R} \$ 35,00$ para os meses 1 e 2 e $R \$ 40,00$ para os meses 3 e 4;

i. esta vacina, sem ter o frasco-ampola aberto, tem uma validade superior aos quatro meses analisados neste artigo.

Nestes quatro meses analisados, o hospital comprava as vacinas de acordo com a demanda do mês, conforme apresentando no Quadro 1, em que também são exibidos os custos mensais e o total do período.

A seguir, utilizando as informações apresentadas do Quadro 1, o modelo de PD foi estruturado. Avaliaram-se as três condições de custos viáveis que ocorram no mês 4, apresentadas no Quadro 2. Estas possibilidades se devem a restrição citada anteriormente, que não deve sobrar estoque de vacinas no fim do último mês, desta forma, o mês pode iniciar com nada em estoque, 5.000 ou 10.000 unidades, ou seja, $f_{4}\left(e_{3}=0, x_{4}\right)$ $f_{4}\left(e_{3}=5.000, x_{4}\right)$ e $f_{4}\left(e_{3}=10.000, x_{4}\right)$. 
Quadro 1

Cenário atual

\begin{tabular}{cccc}
\hline Mês & Estoque inicial do estágio & Quantidade a ser comprada & Custo do estágio \\
\hline 1 & 5.000 & 15.000 & $\mathrm{R} \$ 529.337,50$ \\
2 & 0 & 10.000 & $\mathrm{R} \$ 351.200,00$ \\
3 & 0 & 15.000 & $\mathrm{R} \$ 601.200,00$ \\
4 & 0 & 10.000 & $\mathrm{R} \$ 401.200,00$ \\
& & Custo total: & $\mathbf{R} \mathbf{1 . 8 8 2 . 9 3 7 , 5 0}$ \\
\hline
\end{tabular}

Fonte: Elaborado pelos autores (2019)

\section{Quadro 2}

Resultado do estágio 4

\begin{tabular}{cccc}
\hline & Mês 4 & & \\
\hline Estoque remanescente do estágio anterior & $\begin{array}{c}\text { Quantidade a ser } \\
\text { comprada }\end{array}$ & Custo total do estágio & Estoque final \\
\hline 0 & 10.000 & $\mathrm{R} \$ 401.200,00$ & 0 \\
5.000 & 5.000 & $\mathrm{R} \$ 205.200,00$ & 0 \\
10.000 & 0 & $\mathrm{R} \$ 8.000,00$ & 0 \\
\hline
\end{tabular}

Fonte: Elaborado pelos autores (2019)

No Quadro 2, para calcular as possíveis quantidades de compra, foi utilizada a equação de continuidade de fluxo, conforme a Equação 1. Assim, é possível calcular $x_{4}$, de acordo com as Equações 3 a 5 .

para $e_{3}=0: x_{4}=e_{4}-e_{3}+d_{4}=0-0+10000=10000$

para $e_{3}=5000: x_{4}=e_{4}-e_{3}+d_{4}=0-5000+10000=50000$

para $e_{3}=1000: x_{4}=e_{4}-e_{3}+d_{4}=0-1000+10000=0$

Já o mês 3 pode iniciar com até 25.000 unidades, por ser o somatório das demandas deste e do quarto mês. Para cada possível valor de estoque inicial, há alguns cenários praticáveis, conforme exemplificado no Quadro 3.

Neste exemplo exibido no Quadro 3, todas possibilidades analisadas são as que iniciam o mês sem nada em estoque. Então, é necessário atender a demanda de 15.000 unidades e foi feito um estudo com diferentes possibilidades referentes a quantidade para permanecer em estoque para o mês 4 . O único custo a ser considerado neste cenário é o da compra, já que não há o custo de estoque das vacinas remanescentes.

\section{Quadro 3}

Parte do estudo realizado para o estágio 3

\begin{tabular}{ccccc}
\hline $\begin{array}{c}\text { Estoque remanescente } \\
\text { do estágio anterior }\end{array}$ & $\begin{array}{c}\text { Estoque para } \\
\text { estágio seguinte }\end{array}$ & $\begin{array}{c}\text { Quantidade a ser } \\
\text { comprada }\end{array}$ & $\begin{array}{c}\text { Custo de estoque das } \\
\text { vacinas remanescentes }\end{array}$ & Custo da compra \\
\hline 0 & 0 & 15.000 & $\mathrm{R} \$ 0,00$ & $\mathrm{R} \$ 601.200,00$ \\
& 5.000 & 20.000 & $\mathrm{R} \$ 0,00$ & $\mathrm{R} \$ 801.200,00$ \\
$\mathrm{~N}$ & 10.000 & 25.000 & $\mathrm{R} \$ 0,00$ & $\mathrm{R} \$ 1.001 .200,00$ \\
\hline
\end{tabular}

Fonte: Elaborado pelos autores (2019)

Com esta análise, é percebido que o menor custo é de $R \$ 601.200,00$, a hipótese de não deixar nenhuma vacina em estoque para ser utilizada no mês subsequente. Este estudo foi feito para as demais possíveis quantidades em estoque no início deste mês, isto é, $5.000,10.000,15.000$, 20.000 e 25.000 vacinas. O Quadro 4 apresenta os menores custos destas análises para cada uma das condições iniciais, já que o modelo estruturado visa minimizar os custos. 
Quadro 4

Resultados do estágio 3

\begin{tabular}{cccc}
\hline & Mês 3 & \\
\hline $\begin{array}{c}\text { Estoque remanescente do } \\
\text { estágio anterior }\end{array}$ & Quantidade a ser comprada & Custo total do estágio & $\begin{array}{c}\text { Estoque a ser enviado } \\
\text { para o próximo estágio }\end{array}$ \\
\hline 0 & 15.000 & $\mathrm{R} \$ 601.200,00$ & 0 \\
5.000 & 10.000 & $\mathrm{R} \$ 405.200,00$ & 0 \\
10.000 & 5.000 & $\mathrm{R} \$ 209.200,00$ & 0 \\
15.000 & 5.000 & $\mathrm{R} \$ 213.200,00$ & 5.000 \\
20.000 & 0 & $\mathrm{R} \$ 16.000,00$ & 5.000 \\
25.000 & 0 & $\mathrm{R} \$ 2.000,00$ & 10.000 \\
\hline
\end{tabular}

Fonte: Elaborado pelos autores (2019)

O custo mínimo exibido no Quadro 3 foi calculado pela Equação 6.

$\operatorname{minf}_{3}\left(e_{2}, x_{3}\right)=[$ (custo do estoque do mês 2)+ (custo da compra do mês 3 )]

Já a condição inicial do segundo mês é de estoque inicial $e_{1}$ de até 35.000 unidades, que é o somatório das demandas deste, do terceiro e do quarto mês. Ainda, o máximo que pode ficar em estoque para os meses seguintes $e_{2}$ é 25.000 uni- dades. Então, o Quadro 5 mostra os menores custos de cada análise do segundo mês, considerando estes requisitos.

Já o Quadro 6 apresenta o estudo realizado para o mês 1 . Este mês tem somente uma condição inicial, que é o estoque inicial de 5.000 unidades, conforme descrito nos parâmetros do modelo. Foram analisadas todas possíveis quantidades para ficarem em estoque e serem utilizadas nos meses seguintes, ou seja, até 35.000 vacinas. O menor custo $f_{1}\left(e_{0}=5.000, x_{1}\right)$ calculado está exibido no Quadro 6.

\section{Quadro 5}

Resultados do estágio 2

\begin{tabular}{cccc}
\hline \multicolumn{3}{c}{ Mês 2 } \\
\hline $\begin{array}{c}\text { Estoque remanescente } \\
\text { do estágio anterior }\end{array}$ & $\begin{array}{c}\text { Quantidade a ser } \\
\text { comprada }\end{array}$ & Custo total do estágio & $\begin{array}{c}\text { Estoque a ser enviado para o } \\
\text { próximo estágio }\end{array}$ \\
\hline 0 & 35.000 & $\mathrm{R} \$ 1.226 .200,00$ & 25.000 \\
5.000 & 30.000 & $\mathrm{R} \$ 1.054 .437,50$ & 25.000 \\
10.000 & 25.000 & $\mathrm{R} \$ 882.675,00$ & 25.000 \\
15.000 & 20.000 & $\mathrm{R} \$ 710.912,50$ & 25.000 \\
20.000 & 15.000 & $\mathrm{R} \$ 539.150,00$ & 25.000 \\
25.000 & 10.000 & $\mathrm{R} \$ 367.387,50$ & 25.000 \\
30.000 & 5.000 & $\mathrm{R} \$ 195.625,00$ & 25.000 \\
35.000 & 0 & $\mathrm{R} \$ 22.662,50$ & 25.000 \\
\hline
\end{tabular}

Fonte: Elaborado pelos autores (2019)

\section{Quadro 6}

Resultados do estágio 1

\section{Mês 1}

Estoque inicial Quantidade a ser comprada Custo total do estágio Estoque a ser enviado para o próximo estágio

$5.000 \quad$ R $\$ 529.337,50$

0

Fonte: Elaborado pelos autores (2019) 
Assim, foi obtido o resultado final do modelo de otimização, ou seja, o cenário com o menor cus- to possível, conforme apresentado no Quadro 7, e esquematizado na Figura 4.

\section{Quadro 7}

Resultado final

\begin{tabular}{cccc}
\hline Mês & Estoque inicial do estágio & Quantidade a ser comprada & Custo do estágio \\
\hline 1 & 5.000 & 15.000 & $\mathrm{R} \$ 529.337,50$ \\
2 & 0 & 35.000 & $\mathrm{R} \$ 1.226 .200,00$ \\
3 & 25.000 & 0 & $\mathrm{R} \$ 20.000,00$ \\
4 & 10.000 & 0 & $\mathrm{R} \$ 8.000,00$ \\
& & Custo total & $\mathbf{R} \mathbf{1 . 7 8 3 . 5 3 7 , 5 0}$
\end{tabular}

Fonte: Elaborado pelos autores (2019)

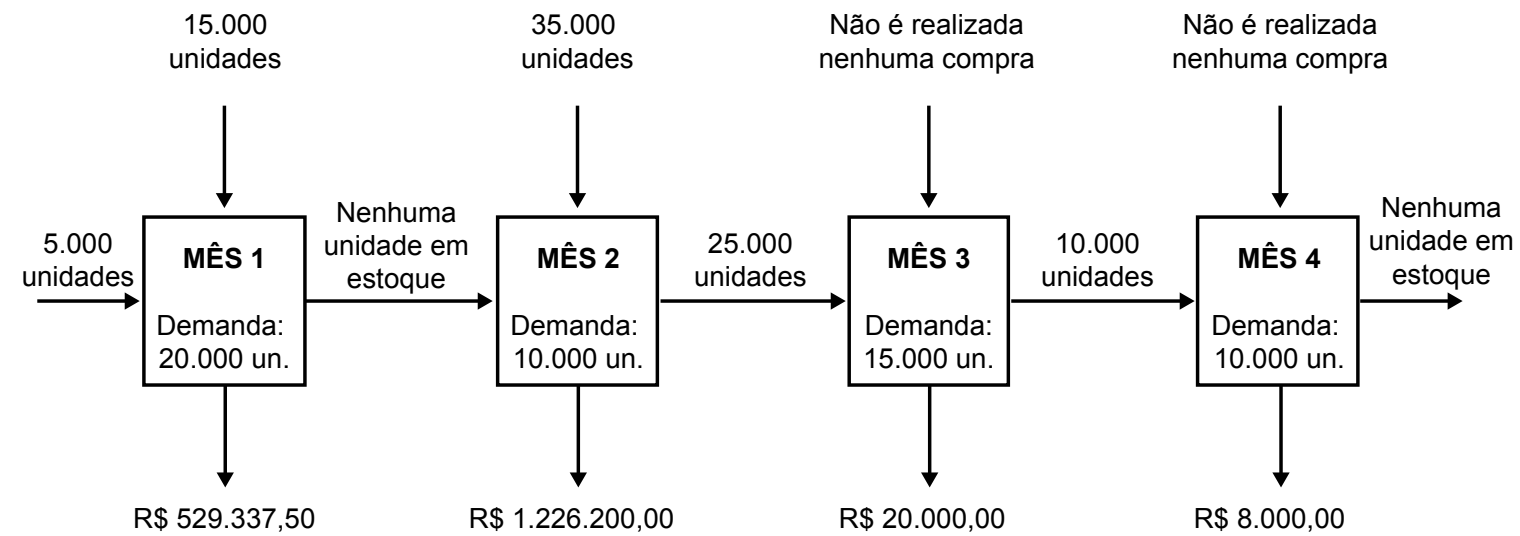

Figura 4 - Representação gráfica do cálculo obtido com PD

Fonte: Elaborado pelos autores (2019)

Então, com o processo de compras otimizado, o custo total foi de $R \$ 1.783 .537,50$, enquanto o hospital gastou $R \$ 1.882 .937,50$ realizando compras mensais na quantidade da demanda necessária para o respectivo mês. Ficou evidente uma redução de custos de $R \$ 99.400,00$, representando uma economia de 5,57\%.

\section{DISCUSSÃO}

Com este trabalho, ficou evidente a contribuição na utilização de um modelo matemático para auxiliar a gestão de verbas da saúde pública ou mesmo privada, ao passo que hospitais podem ter seus processos administrativos, de finanças e logística ocorrendo de forma otimizada, utilizando de uma maneira mais eficiente os recursos escassos ${ }^{2}$, podendo então utilizar esta verba excedente em melhor atendimento aos pacientes ou financiando novas pesquisas ${ }^{14,15}$.

Este artigo estudou o conceito de Programação Dinâmica aplicado a minimização de custos de compras de vacinas em um hospital presente na região sul do país. Utilizando as informações fornecidas pelo hospital, observou-se que o menor custo possível foi de $\mathrm{R} \$ 1.783 .537,50$ para os quatro meses analisados. Este valor é referente a uma compra no primeiro mês de 15.000 unidades e outra de 35.000 no segundo mês, e somando os custos de estoque. Dando continuidade neste estudo multidisciplinar, foi comparado o resultado obtido com a Programação Dinâmica com a situação atual do hospital, evidenciando uma possível economia de $5,57 \%$ com o uso da PD, que representa quase $R \$ 100.000,00$.

Informações como esta podem ser vantajosas para os gestores hospitalares, uma vez que 
o conhecimento desta técnica pode auxiliar na tomada de decisão da maneira que será realizada a compra dos medicamentos. Assim, este trabalho teve como objetivo instruir o uso de Programação Dinâmica e suas possíveis aplicações práticas, possibilitando que os processos administrativos, de finanças e logística ocorram de forma otimizada. O modelo proposto resolve adequadamente o processo analisado, e desta forma, este artigo alcançou seus objetivos satisfatoriamente, podendo o modelo apresentado ser aplicado em casos similares.

\section{REFERÊNCIAS}

1. Duarte NCM, Bitar JPS, Miglioli JP, Pereira MM, Yodono NBP, Araújo $T R$, et al. Gestão de compras em um hospital de ensino terciário: um estudo de caso. Medicina (Ribeirão Preto, Online). 2015 [acesso em 01 nov 2019]; 48(1):48-56. Disponível em: http://www.revistas.usp. $\mathrm{br} / \mathrm{rmrp} / \mathrm{article} / \mathrm{view} / 96932$

2. Dallora MELV, Forster AC. A importância da gestão de custos em hospitais de ensino: considerações teóricas. Medicina (Ribeirão Preto, Online). 2008 [acesso em 01 nov 2019]; 41(2):135-42. Disponível em: http://www.revistas.usp. $\mathrm{br} / \mathrm{rmrp} /$ article/view/259

3. Gerlack LF, Karnikowski MGO, Areda CA, Galato D, Oliveira AG, Álvares J, et al. Gestão da assistência farmacêutica na atenção primária no Brasil. Rev Saúde Pública. 2017 [acesso em 01 nov 2019];51(Supl.2):15s. Disponível em: http://www.rsp.fsp.usp.br/artigo/gestao-da-assistencia-farmaceutica-na-atencao-primaria-no-brasil/

4. Nascimento RCRM, Álvares J, Guerra Junior AA, Gomes IC, Costa EA, Leite SN, et al. Disponibilidade de medicamentos essenciais na atenção primária do Sistema Único de Saúde. Rev Saúde Pública. 2017 [acesso em 01 nov 2019];51(Supl.2):10s. Disponível em: http://www.scielo.br/scielo.php?script=sci_arttext\&pi$\mathrm{d}=$ S0034-89102017000300303\&Ing=en\&nrm=iso\&tIng=pt

5. Abbas K. Gestão de custos em organizações hospitalares. [Dissertação de Mestrado em Engenharia de Produção] Universidade Federal de Santa Catarina; 2001.
6. Raupp FM, Gibbon ARO, Beuren IM. O uso da programação dinâmica pela contabilidade de custos na otimização de recursos escassos. Contabilidade Vista \& Revista. 2004;15(1):73-89.

7. Scarcelli ROC. Programação dinâmica aplicada à otimização individualizada e desacoplada das usinas hidrelétricas de sistemas hidrotérmicos. [Tese de Doutorado]. Universidade de São Paulo; 2012.

8. Wang C, Yu C, Tang S, Xiao J, Sun J, Meng X. A general and fast distributed system for large-scale dynamic programming applications. Parallel Computing. 2016;60:1-21.

9. Diban P, Aziz M, Foo D, Jia X, Li Z, Tan R. Optimal biomass plantation replanting policy using dynamic programming. Journal of Cleaner Production. 2016;126:409-18.

10. Zhang $X$, Wu $C$, Zuo L. Minimizing fuel consumption of a gas pipeline in transient states by dynamic programming. Journal of Natural Gas Science and Engineering. 2016;28:193-203. Disponível em: https://www.sciencedirect.com/science/article/abs/pii/ S1875510015302754

11. Duchaud JL, Notton G, Darras C, Voyant C. Power ramp-rate control algorithm with optimal State of Charge reference via Dynamic Programming. Energy. 2018;149:709-17. Disponível em: https://www.sciencedirect.com/science/article/abs/pii/S0360544218302925

12. Colin EC. Pesquisa Operacional: 170 aplicações em estratégia, finanças, logística, produção, marketing e vendas. Rio de Janeiro: LTC, 2007.

13. Hillier F, Lieberman G. Introduction to Operations Research. 8. ed. Nova Iorque: McGraw-Hill, 2005.

14. Barros R, Costa E, Santos D, Souza G, Álvares J, Guerra Junior $A$, et al. Acesso a medicamentos: relações com a institucionalização da assistência farmacêutica. Rev Saúde Pública. 2017;51(Supl.2):8s. Disponível em: http://www.scielo.br/scielo.php?script=sci_abstract\&pi$\mathrm{d}=$ S0034-89102017000300317\&lng=en\&nrm=iso\&tlng=pt

15 Faleiros D, Acurcio F, Álvares J, Nascimento R, Costa E, Guibu I, et al. Financiamento da assistência farmacêutica na gestão municipal do Sistema Único de Saúde. Rev Saúde Pública. 2017;51(Supl.2):14s. Disponível em: http://www.scielo.br/scielo.php?pid=S0034=89102017000300302-\&scriptsci_arttext\&tlng=pt 